\title{
Nanoscale
}

\section{A light-driven supramolecular nanowire actuator $\dagger$}

Cite this: Nanoscale, 2015, 7, 6457

Received 16th February 2015,

Accepted 13th March 2015

DOI: $10.1039 / c 5 n r 01118 c$

www.rsc.org/nanoscale

A single photomechanical supramolecular nanowire actuator with an azobenzene-containing 1,3,5-tricarboxamide derivative is developed by employing a direct writing method. Single nanowires display photoinduced reversible bending and the bending behavior follows first-order kinetics associated with azobenzene photoisomerization. A wireless photomechanical nanowire tweezers that remotely manipulates a single micro-particle is also demonstrated.

Photomechanical actuators, ${ }^{1-6}$ which convert light energy directly into physical motion, have clear advantages over conventional electrostatic actuators ${ }^{7,8}$ because they can be remotely controlled in the absence of invasive wires or electrodes. An additionally attractive feature is that actuation of photomechanical actuators can be readily tuned through control of the wavelength, direction and intensity of input light. Owing to these properties, a wide variety of photoresponsive actuating materials and systems, including photochromic crystals, ${ }^{1,6,9-11}$ azobenzene derivatives, ${ }^{2,5,12,36}$ and supramolecular hydrogel, ${ }^{3}$ have been developed thus far. However, the development of photomechanical nanowire actuators, which are in critical demand because of their use as active actuating components in nanodevices, has been for the most part unexplored. In this study, we developed a single photomechanical molecular nanowire actuator that employs an azobenzene-containing supramolecule as a supramolecular nanowire scaffold. Single photomechanical nanowires undergo bending upon

\footnotetext{
${ }^{a} X$-ray Imaging Center, Department of Materials Science and Engineering, Pohang University of Science and Technology, Pohang 790-784, Republic of Korea. E-mail: jhje@postech.ac.kr

${ }^{b}$ Department of Chemical Engineering, Hanyang University, Seoul 133-791, Republic of Korea

${ }^{c}$ Institute of Nano Science and Technology, Hanyang University, Seoul 133-791, Republic of Korea. E-mail: jmk@hanyang.ac.kr

$\dagger$ Electronic supplementary information (ESI) available: Experimental details, X-ray powder diffraction (XRD) patterns of solution-crystallized sample, meniscus-guided microwires, and freeze-dried sample of Azo-1, Schematic of experimental set-up, 3D bending motion of Azo-1 nanowire, FE-SEM image of a bent Azo-1 nanowire after UV irradiation, real-time grazing incidence X-ray diffraction (GIXD) for an Azo-1 microwire, Imaging analyses, Absorption spectra of an Azo-1 film, and thermostability of Azo-1 nanowire. See DOI: 10.1039/c5nr01118c
}

irradiation with UV light that is reversed upon visible light irradiation, and the bending behavior follows first-order kinetics associated with azobenzene photoisomerization. A wireless photomechanical nanowire tweezers that remotely manipulates a single micro-particle was also developed. The strategy employed to design the single photomechanical nanowires could very well serve as the foundation for the development of other novel molecular based light-driven nanowire actuators.

Photoinduced three-dimensional (3D) motion comprised of bending, ${ }^{13}$ twisting, ${ }^{14,15}$ and rotation ${ }^{16}$ has highly promising practical applications. In particular, one-dimensional (1D)-like photomechanical actuators are beneficial to $3 \mathrm{D}$ motion due to its high degree of freedom motion. ${ }^{17}$ The development of photomechanical nanowire actuators are in critical demand for their use as active actuating components in nanodevices. However, current approaches, such as recrystallization, ${ }^{1,6,9-11,13-15}$ inkjet printing, ${ }^{4}$ melt-and-draw method ${ }^{17,18}$ only afford micron-sized actuators and they suffer from the lack of dimensional control. Irie et al. ${ }^{19}$ (or Ikeda et $a .^{12}$ ) demonstrated photo-responsive nanoactuating in a bulk crystal (or nanoparticles), which was, however, restricted to one-dimension and showed very limited actuating displacements $(<10 \mathrm{~nm})$. The template-assisted solvent annealing method ${ }^{20,21}$ by Al-Kaysi et al. provided photomechanical nanowires $(\sim 35 \mathrm{~nm})$, which were randomly dispersed on a substrate and therefore showed a motion limitation in two-dimensions. Accordingly, a reliable method for photomechanical nanowire actuators with omnidirectional actuating in nanoscale and large $(>\mu \mathrm{m})$ actuating displacements has yet to be developed.

Herein we report a new single photomechanical nanowire actuator that shows three-dimensional (3D) actuating in nanoscale for the first time, by vertically growing the azobenzene containing supramolecule, tris(4-((E)-phenyldiazenyl)phenyl)benzene-1,3,5-tricarboxamide (Azo-1) (Fig. 1(a)). A meniscusguided solidification method, ${ }^{21}$ which has proven to be an effective bottom-up strategy for organic nanowires, was employed. The key feature of the method is that it relies on confined solidification of an organic solution within a nanoscale meniscus (Fig. 1(b)). Single vertical nanowires can be 
a

generated at room temperature by guiding the meniscus upward using a micropipette. The azobenzene containing benzenetricarboxamide (BTC) derivative, Azo-1, was selected as the nanowire material owing to its photoresponsive shape change property as well as its intriguing $C_{3}$-symmetrical structure that enables facile formation of columnar supramolecules. ${ }^{26}$ The Azo-1 nanowires fabricated by the meniscus-guided method have a monoclinic crystal structure ${ }^{26}$ (Fig. S1, ESI $\dagger$ ). In general, it is very difficult to control the sizes and shapes of 1D nanostructures of self-assembled supramolecules. ${ }^{27}$ In contrast, owing to its direct writing capability using organic materials, the meniscus-guided solidification method enables ready growth of 1D nanostructure..$^{22-25}$ In fact, this approach can be utilized to grow vertical Azo-1 nanowires that have smooth surfaces in high-aspect-ratios and diameters $(d)$ in the range of 200 to $1000 \mathrm{~nm}$ that are pulling speed governed (Fig. 1(c)). Furthermore, 3D writing ability and tunable pulling time property of the method enables exact positioning and alignment of individual Azo-1 nanowires, as demonstrated by the precisely controlled formation of the letters ' $\mathrm{B}$ ', ' $\mathrm{T}$ ', and ' $\mathrm{C}$ ' comprised of nanowire arrays with respective lengths of $20,15,10 \mu \mathrm{m}$ (Fig. 1(d)).

Importantly, vertically grown Azo-1 nanowires undergo reversible photo-induced mechanical motion (Fig. S2 in ESI $\dagger$ for experimental set-up). This is demonstrated by the observation that bending and unbending of a single Azo-1 nanowire $(d \sim 500 \mathrm{~nm}$ and $l$ (length) $\sim 20 \mu \mathrm{m}$ ) take place upon respective

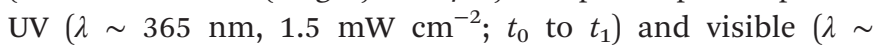
$455 \mathrm{~nm}, 4 \mathrm{~mW} \mathrm{~cm}^{-2} ; t_{1}$ to $t_{2}$ ) light irradiation (Fig. 2(a)). Upon a

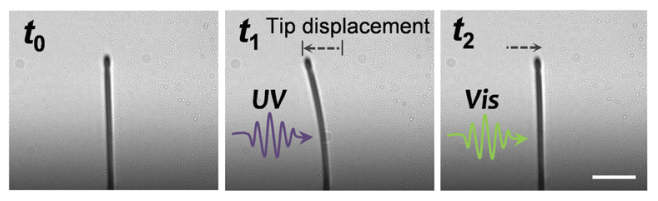

b

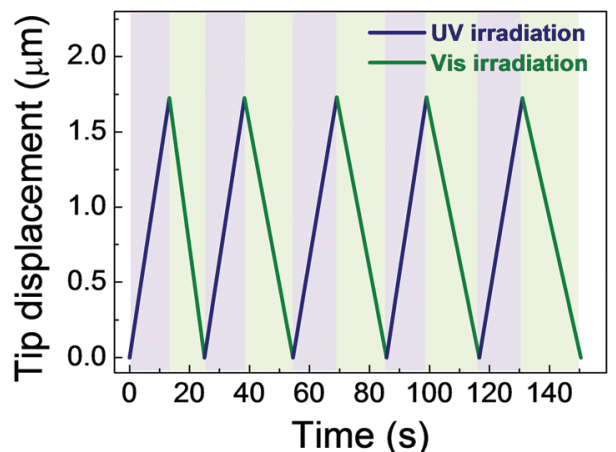

C

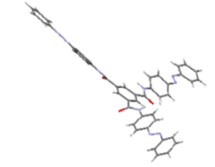

Trans-Azo-1

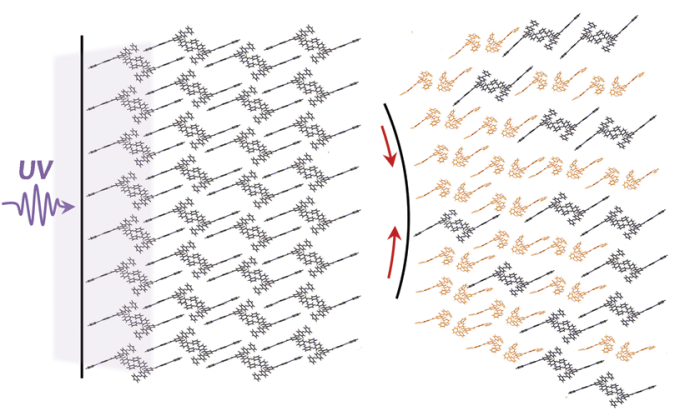

Fig. 2 (a) Optical microscope images show photo-induced bending $\left(t_{\circ} \rightarrow t_{1}\right)$ and unbending $\left(t_{1} \rightarrow t_{2}\right)$ of an Azo-1 nanowire $(500 \mathrm{~nm}(d) \times$ $22.5 \mu \mathrm{m}(l))$ upon respective UV $\left(\lambda \sim 365 \mathrm{~nm}, 1.5 \mathrm{~mW} \mathrm{~cm}^{-2}\right)$ and visible $\left(\lambda \sim 455 \mathrm{~nm}, 4 \mathrm{~mW} \mathrm{~cm}{ }^{-2}\right)$ light irradiation. Scale bar, $10 \mu \mathrm{m}$. (b) A plot of the tip displacement as a function of time showing the reversible photoinduced bending and unbending process of the Azo-1 nanowire by alternating the UV (purple) and visible (green) light irradiation. (c) Schematic of trans-cis photoisomerization of Azo-1 in the crystalline nanowire.

UV light irradiation, a single nanowire bends toward the light source and returns to its initial position upon visible light irradiation from the same direction. Importantly, single nanowires display reversible $3 \mathrm{D}$ bending motion depending on the position of the UV and visible light sources (ESI Movie S1 and Fig. S3 in ESI $\dagger$ ). Finally, the reversible bending process takes place repeatedly by alternating the UV and visible light irradiation without fragmentation (Fig. 2(b), see also ESI Movie $\mathrm{S} 2$, and Fig. S4 in $\mathrm{ESI}_{\dagger}^{\dagger}$ ).

The bending phenomenon experienced by the nanowire $(d \sim 500 \mathrm{~nm})$ is likely a consequence of photoisomerization about the azo $\pi$-bond in the component Azo- 1 supramolecules (Fig. 2(c)). ${ }^{28}$ UV light irradiation of azobenzenes typically 
a

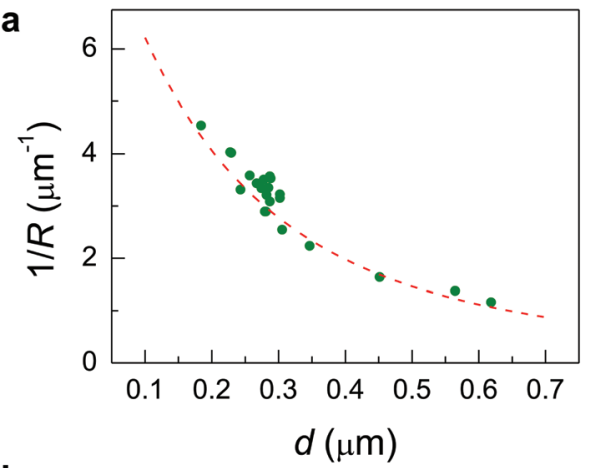

b

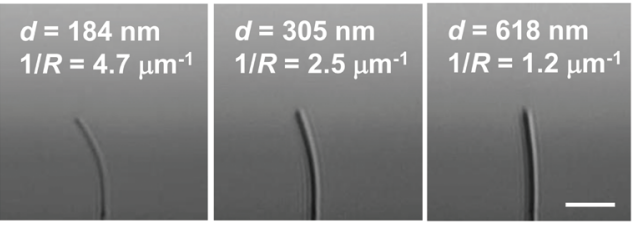

Fig. 3 (a) The bending curvature $(1 / R ; R=$ the bending radius) of the Azo-1 nanowire, measured as a function of $d$ after UV light irradiation for $10 \mathrm{~s}$. The decrease in $1 / R$ with $d$ (closed green circles) is well matched with a theoretical model (dashed red line), $1 / R \sim \lambda / w^{2}[(\lambda / w+$ $\left.0.5) \mathrm{e}^{-w / L}-\lambda / w+0.5\right],{ }^{30}$ where $w$ is the thickness of a solid plate and $\lambda$ is the attenuation length, when $\lambda$ is $100 \mathrm{~nm}$. This indicates that the length contraction takes place at the surface of the nanowire. The depth profile of the photoisomerization is described by $c_{c i s}(x)=c_{c i s}(0) \times\{\exp (-x / \lambda)\}$, where $c_{c i s}$ is the cis fraction of Azo-1 supramolecules and $\lambda$ is the attenuation length, based on the theoretical model by Beer's law. ${ }^{30}$ (b) Representative optical microscope images of the nanowires (l (length) $=$ $22.5 \mu \mathrm{m}$ ) with different diameters after UV light irradiation for $10 \mathrm{~s}$. Scale bar, $10 \mu \mathrm{m}$.

promotes trans-to-cis isomerization, a chemical process that is accompanied by a significant decrease in the overall length of the molecule from $c a .9 .0 \AA$ in the trans form to $c a .5 .5 \AA$ in the cis form. The molecular geometry change caused by the photoisomerization reaction is known cause a macroscopic shape change. ${ }^{2}$ For instance, polymer networks, cross-linked with azobenzene moieties, undergo contraction when irradiated with UV light. ${ }^{29}$

It should be noted that absorption of $365 \mathrm{~nm}$ UV light by the Azo-1 nanowire occurs only within $\sim 100 \mathrm{~nm}$ from the surface of the nanowire, as estimated by fitting a theoretical model (dashed red line) for photoinduced bending based on Beer's law $^{30}$ (Fig. 3(a)). Here the bending curvature $(1 / R ; R=$ the bending radius) of the Azo-1 nanowire was measured as a function of $d$ after irradiation with UV light for $10 \mathrm{~s} \mathrm{(Fig.} \mathrm{3(b)).}$ The small attenuation length $(\sim 100 \mathrm{~nm})$, compared to that of liquid-crystal elastomer $(\sim 1 \mu \mathrm{m}),{ }^{31,32}$ is attributed mostly to high density of the azobenzene chromophores. ${ }^{26}$ In fact, we experimentally confirmed the contraction of the Azo-1 nanowire at the surface region $(<100 \mathrm{~nm})$ upon UV irradiation, based on using a real-time grazing incidence X-ray diffraction (GIXD) method (Fig. S5, ESI $†$ ). Thus, the length contraction that takes place at the surface of the nanowire is responsible for the photoinduced bending (Fig. 4(a)). a

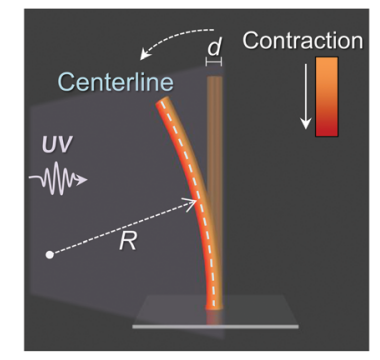

c

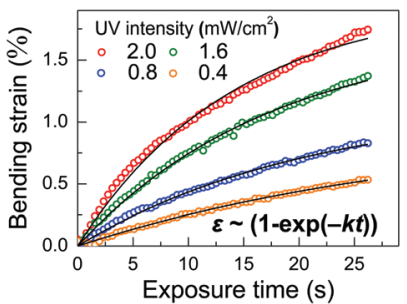

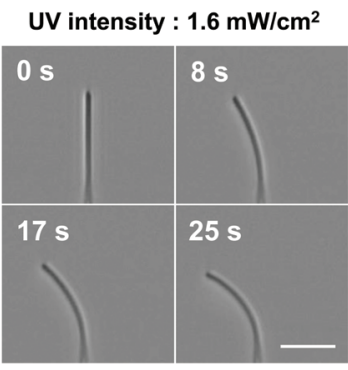

d

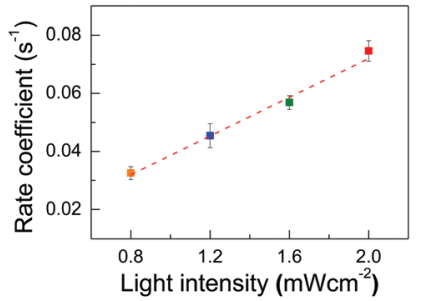

Fig. 4 (a) Schematic illustration of the photoinduced bending of an Azo-1 nanowire upon UV light irradiation. The bending strain is $\varepsilon=(d / 2) /$ $R$, where $d$ is the diameter of an Azo-1 nanowire and $R$ is the bending radius. (b) Optical microscope images showing bending of an Azo-1 nanowire $(450 \mathrm{~nm}(d) \times 22.5 \mu \mathrm{m}(l))$ promoted by a given UV intensity in real-time. Scale bar, $10 \mu \mathrm{m}$. (c) Plots of bending strains as a function of exposure time for various UV intensities. Bending strain exponentially increases with exposure time in accord with the relationship 1 - exp$(-k t)$, where $k$ is the rate coefficient. The coefficients of determination $\left(R^{2}\right)$ are $0.987\left(2.0 \mathrm{~mW} \mathrm{~cm}{ }^{-2}\right), 0.993\left(1.6 \mathrm{~mW} \mathrm{~cm}^{-2}\right), 0.995(1.2 \mathrm{~mW}$ $\left.\mathrm{cm}^{-2}\right)$, and $0.992\left(0.8 \mathrm{~mW} \mathrm{~cm}^{-2}\right)$. (d) The rate coefficient $(k)$, estimated from fitting the strain data in (c), is linearly proportional to the UV light intensity $\left(I_{0}\right)$. A dashed red line is a linear fit.

In order to gain quantitative information on the bending behavior of the Azo-1 nanowires, the kinetics of the photoinduced bending were investigated. Specifically, the bending of an Azo-1 nanowire $(d \sim 450 \mathrm{~nm})$ promoted by a given UV intensity was determined with time (Fig. 4(b)). The bending strain $(\varepsilon=(d / 2) / R)$ of the Azo-1 nanowire was then estimated by using the bending radius $R$ (Fig. 4(a)). By taking advantage of an image-processing and MATLAB-based circle fitting algorithm, the bending curvatures $(1 / R)$ of a nanowire at various exposure times were calculated (Fig. S6, ESI $\dagger$ ). The results show that bending strain exponentially increases with exposure time in accord with the relationship $1-\exp (-k t)$, where $k$ is the rate coefficient (Fig. 4(c)). The time dependence of the strain corresponds to first-order kinetics of the trans $\rightarrow$ cis photoisomerization in azobenzene, $\left(c_{c i s} \propto[1-\exp (-k t)]\right)$, where $c_{c i s}$ is the $c i s$ fraction of chromophores (Fig. S7, ESI $\dagger$ ). The bending strain and the degree of photoisomerization ([1 $\left.\left.\exp \left(-k_{\text {react }} t\right)\right] \times 100(\%)\right)$ showed almost a linear relationship (Fig. S7(c), ESI $\dagger$ ), as reported..$^{33}$ The result represents that the bending motion of the Azo-1 nanowire is directly linked to the molecular level transition of the azobenzene stereoisomeric forms. The rate coefficient ( $k$ ) (Fig. 4(d)), estimated from fitting the strain data based on $\varepsilon \sim(1-\exp (-k t))$ given in Fig. $4(\mathrm{c})$, is linearly proportional to the UV light intensity $\left(I_{0}\right)$. 
This finding confirms that the kinetics of the photoinduced bending of the nanowires parallels the first-order kinetics of the azobenzene photoisomerization process. It is noteworthy that irradiation using a low intensity $\left(0.8 \mathrm{~mW} \mathrm{~cm}^{-2}\right)$ of UV light for a few seconds is sufficient to induce bending of the nanowires, (Fig. 4(c)). This light intensity level meets the accepted safety standard. ${ }^{34}$ (ACGIH 2005, $1.0 \mathrm{~mW} \mathrm{~cm}{ }^{-2}$ for periods lasting $<1000 \mathrm{~s}$ ), suggesting that the system is potentially suitable for use in biomedical devices.

Remarkably, the Azo-1 nanowire actuators display a very high thermal stability. The bending strains of the Azo-1 nanowires, measured as a function of time at a given temperature $\left(25,60\right.$, and $\left.90{ }^{\circ} \mathrm{C}\right)$ after irradiation with UV light $(1.6 \mathrm{~mW}$ $\mathrm{cm}^{-2}, 10 \mathrm{~s}$ ), display little variations up to one month (Fig. S8, ESI $\dagger)$. This high thermal stability is attributed to the fact that the rate of the thermal back-isomerization (cis $\rightarrow$ trans) reaction of the Azo-1 supramolecules is exceptionally low, as a consequence presumably of strong intermolecular interactions between the cis forms.

The final phase of current investigation focused on a demonstration that the photo-induced bending phenomenon could be used to design a photomechanical nanowire tweezers, which is controlled remotely without the need for invasive wires or electrodes that are required in conventional electrostatic actuators. ${ }^{7,8}$ As illustrated schematically in Fig. 5, bending of an Azo-1 nanowire actuator arm (yellow) against a fixed polystyrene (PS) nanowire arm (black), the latter of which does not experience a photomechanical response, would allow the device to be employed to grip a micro-object (gray sphere) upon UV light irradiation. To explore this design strategy, both

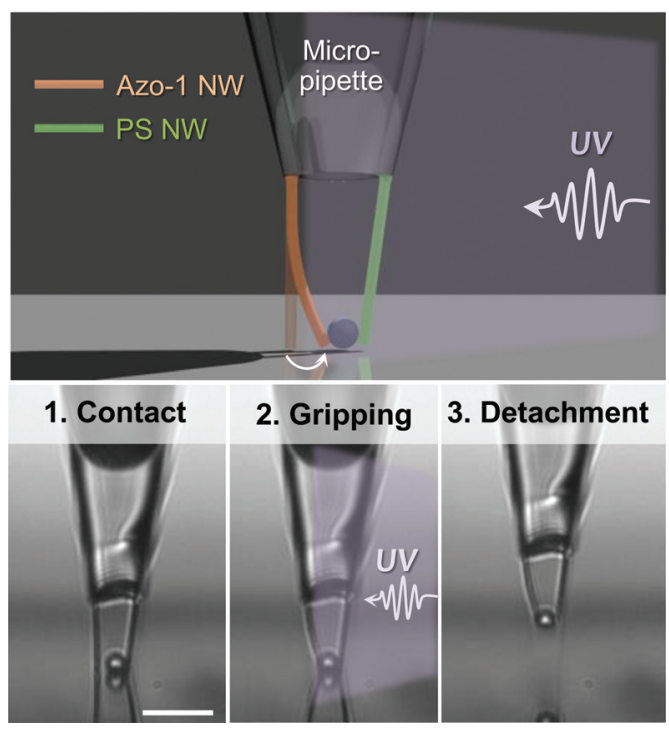

Fig. 5 Azo-1 and a PS nanowire $(500 \mathrm{~nm}(d) \times 12.5 \mu \mathrm{m}(l))$ arms were individually integrated on the tip of a glass microcapillary tube. As demonstrated by the optical microscope images, the resulting tweezers successfully grips a PS microparticle $(d \sim 4 \mu \mathrm{m})$ on a silicon substrate upon irradiation with UV light $\left(1.5 \mathrm{~mW} \mathrm{~cm}^{-2}\right.$ for $\left.20 \mathrm{~s}\right): 1$ (contact) $\rightarrow$ 2 (gripping) $\rightarrow 3$ (detachment). Scale bar, $20 \mu \mathrm{m}$.
Azo-1 $(d \sim 500 \mathrm{~nm})$ and PS nanowire $(d \sim 500 \mathrm{~nm})$ arms were individually integrated on the tip of a glass microcapillary tube using the meniscus-guided method. The resulting tweezers, in fact, was successfully manipulated to pick, lift, and move a PS microparticle $(d \sim 4 \mu \mathrm{m})$ on a silicon substrate upon irradiation with UV light $\left(1.5 \mathrm{~mW} \mathrm{~cm}^{-2}\right.$ for $\left.20 \mathrm{~s}\right)(1 \rightarrow 2 \rightarrow 3$ in Fig. 5) (ESI Movie S2 $\dagger$ ). Based on the low UV dose required to activate the nanowire arm, we anticipate that the new photomechanical tweezers is applicable to biomechanical systems.

\section{Conclusions}

In conclusion, the effort described above led to the development of a method to fabricate a single photomechanical nanowire actuator, which relies on the use of a technique in which solidification of Azo-1 supramolecules takes place within a nanoscale meniscus. Single Azo-1 nanowires were observed to exhibit reversible omnidirectional bending and unbending with large actuating displacements $(\leq 1.7 \mu \mathrm{m})$ when alternately irradiated with UV and visible light. In addition, bending of the photomechanical nanowires displays high thermal stability. The results of the studies also reveal that the bending behavior follows first-order kinetics associated with an azobenzene photoisomerization reaction, ${ }^{35}$ implying that bending is linked to the molecular level phase transition of Azo-1 molecules (trans-cis isomerization of azobenzene). Finally, we have constructed a new photomechanical nanowire tweezers, designed using this novel photoactuation process, and have shown that it can be used to manipulate a single micro-particle remotely. Significantly, the strong possibility exists that light-driven nanowire actuators of the type described above might serve as new nanorobotic devices for manipulation of micro/nano-objects that have general utility in biomedical science and engineering.

\section{Acknowledgements}

This work was supported by the National Research Foundation of Korea (NRF) grant funded by the Korea government (MSIP) (no. 2006-0050683 and 2014R1A2A1A01005862) and by Brain Korea 21 PLUS project for Center for Creative Industrial Materials.

\section{Notes and references}

1 S. Kobatake, S. Takami, H. Muto, T. Ishikawa and M. Irie, Nature, 2007, 446, 778-781.

2 H. Yu and T. Ikeda, Adv. Mater., 2011, 23, 2149-2180.

3 Y. Takashima, S. Hatanaka, M. Otsubo, M. Nakahata, T. Kakuta, A. Hashidzume, H. Yamaguchi and A. Harada, Nat. Commun., 2012, 3, 1270.

4 C. L. van Oosten, C. W. M. Bastiaansen and D. J. Broer, Nat. Mater., 2009, 8, 677-682. 
5 Z. Jiang, M. Xu, F. Li and Y. Yu, J. Am. Chem. Soc., 2013, 135, 16446-16453.

6 O. S. Bushuyev, T. A. Singleton and C. J. Barrett, Adv. Mater., 2013, 25, 1796-1800.

7 O. Kim, T. J. Shin and M. J. Park, Nat. Commun., 2013, 4, 2208.

8 E. Detsi, P. Onck and J. T. M. De Hosson, ACS Nano, 2013, 7, 4299-4306.

9 H. Koshima, R. Matsuo, M. Matsudomi, Y. Uemura and M. Shiro, Cryst. Growth Des., 2013, 13, 4330-4337.

10 F. Terao, M. Morimoto and M. Irie, Angew. Chem., Int. Ed., 2012, 51, 901-904.

11 P. Naumov, J. Kowalik, K. M. Solntsev, A. Baldridge, J.-S. Moon, C. Kranz and L. M. Tolbert, J. Am. Chem. Soc., 2010, 132, 5845-5857.

12 S. Tsoi, J. Zhou, C. Spillmann, J. Naciri, T. Ikeda and B. Ratna, Macromol. Chem. Phys., 2013, 214, 734-741.

13 M. Morimoto and M. Irie, J. Am. Chem. Soc., 2010, 132, 14172-14178.

14 D. Kitagawa, H. Nishi and S. Kobatake, Angew. Chem., Int. Ed., 2013, 52, 9320-9322.

15 L. Zhu, R. O. Al-Kaysi and C. J. Bardeen, J. Am. Chem. Soc., 2011, 133, 12569-12575.

16 M. Yamada, M. Kondo, J.-I. Mamiya, Y. Yu, M. Kinoshita, C. J. Barrett and T. Ikeda, Angew. Chem., Int. Ed., 2008, 47, 4986-4988.

17 T. Yoshino, M. Kondo, J.-I. Mamiya, M. Kinoshita, Y. Yu and T. Ikeda, Adv. Mater., 2010, 22, 1361-1363.

18 W. Deng, M.-H. Li, X. Wang and P. Keller, Liq. Cryst., 2009, 36, 1023-1029.

19 M. Irie, S. Kobatake and M. Horichi, Science, 2001, 291, 1769-1772.

20 R. O. Al-Kaysi, A. M. Müller and C. J. Bardeen, J. Am. Chem. Soc., 2006, 128, 15938-15939.
21 R. O. Al-Kaysi and C. J. Bardeen, Adv. Mater., 2007, 19, 1276-1280.

22 J. T. Kim, S. K. Seol, J. Pyo, J. S. Lee, J. H. Je and G. Margaritondo, Adv. Mater., 2011, 23, 1968-1970.

23 J. Yoo, J. Pyo and J. H. Je, Nanoscale, 2014, 6, 3557-3560.

24 J. Pyo, J. T. Kim, J. Yoo and J. H. Je, Nanoscale, 2014, 6, 5620-5623.

25 J. Yoo, S. Jeong, S. Kim and J. H. Je, Adv. Mater., 2015, DOI: 10.1002/adma.201404945.

26 S. Lee, S. Oh, J. Lee, Y. Malpani, Y.-S. Jung, B. Kang, J. Y. Lee, K. Ozasa, T. Isoshima, S. Y. Lee, M. Hara, D. Hashizume and J.-M. Kim, Langmuir, 2013, 29, 5869-5877.

27 L. C. Palmer and S. I. Stupp, Acc. Chem. Res., 2008, 41, 1674-1684.

28 C. J. Barrett, J.-I. Mamiya, K. G. Yager and T. Ikeda, Soft Matter, 2007, 3, 1249-1261.

29 C. D. Eisenbach, Polymer, 1980, 21, 1175-1179.

30 M. Warner and L. Mahadevan, Phys. Rev. Lett., 2004, 92, 134302.

31 M. Petr and P. T. Hammond, Macromolecules, 2011, 44, 8880-8885.

32 M. Saphiannikova and D. Neher, J. Phys. Chem. B, 2005, 109, 19428-19436.

33 T. Kim, L. Zhu, L. J. Mueller and C. J. Bardeen, J. Am. Chem. Soc., 2014, 136, 6617-6625.

34 American Conference of Governmental Industrial Hygienists (ACGIH). (2005). TLVs and BEIs based on the documentation of the threshold limit values for chemical substances and physical agents and biological exposure indices. Cincinnati: author.

35 I. Mita, K. Horie and K. Hirao, Macromolecules, 1989, 22, 558-563.

36 T. Muraoka, K. Kinbara, Y. Kobayashi and T. Aida, J. Am. Chem. Soc., 2003, 125, 5612-5613. 